\title{
BMJ Open Psychometric properties and use of the DEMQOL suite of instruments in research: a systematic review protocol
}

\author{
Matthias Hoben (D) , ${ }^{1}$ Stephanie A Chamberlain, ${ }^{2}$ Hannah M O'Rourke, ${ }^{1}$ \\ Brittany Elliott, ${ }^{1}$ Shovana Shrestha, ${ }^{1}$ Rashmi Devkota, ${ }^{1}$ Trina Thorne, ${ }^{1}$ Jenny Lam, ${ }^{1}$ \\ Sube Banerjee, ${ }^{3}$ Laura Hughes (1) , ${ }^{4}$ Carole A Estabrooks ${ }^{1}$
}

To cite: Hoben M,

Chamberlain SA, O'Rourke HM et al. Psychometric properties and use of the DEMQOL suite of instruments in research: a systematic review protocol. BMJ Open 2021;11:e041318. doi:10.1136/ bmjopen-2020-041318

- Prepublication history and additional material for this paper is available online. To view these files, please visit the journal online (http://dx.doi.org/10. 1136/bmjopen-2020-041318)

Received 04 June 2020 Revised 20 0ctober 2020 Accepted 18 November 2020

Check for updates

(c) Author(s) (or their employer(s)) 2021. Re-use permitted under CC BY-NC. No commercial re-use. See rights and permissions. Published by BMJ.

${ }^{1}$ Faculty of Nursing, University of Alberta, Edmonton, Alberta, Canada

${ }^{2}$ Faculty of Medicine and Dentistry, University of Alberta, Edmonton, Alberta, Canada

${ }^{3}$ Faculty of Health and Human

Sciences, University of

Plymouth, Plymouth, UK

${ }^{4}$ Centre for Dementia Studies, Brighton and Sussex Medical School, Brighton, UK

Correspondence to Dr Matthias Hoben; mhoben@ualberta.ca

\section{ABSTRACT}

Introduction Dementia is a public health issue and a major risk factor for poor quality of life among older adults. In the absence of a cure, enhancing health-related quality of life (HRQOL) of people with dementia is the primary goal of care. Robust measurement of $\mathrm{HRQOL}$ is a prerequisite to effective improvement. The DEMQOL suite of instruments is considered among the best available to measure HRQoL in people with dementia; however, no review has systematically and comprehensively examined the use of the DEMQOL in research and summarised evidence to determine its feasibility, acceptability and appropriateness for use in research and practice.

Methods and analysis We will systematically search 12 electronic databases and reference lists of all included studies. We will include systematically conducted reviews, as well as, quantitative and qualitative research studies that report on the development, validation or use in research studies of any of the DEMQOL instruments. Two reviewers will independently screen all studies for eligibility, and assess the quality of each included study using one of four validated checklists appropriate for different study designs. Discrepancies at all stages of the review will be resolved by consensus. We will use descriptive statistics (frequencies, proportions, ranges), content analysis of narrative data and vote counting (for the measures of association) to summarise the data elements. Using narrative synthesis, we will summarise what is known about the development, validation, feasibility, acceptability, appropriateness and use of the DEMQOL. Our review methods will follow the reporting and conduct guidelines of the Cochrane Handbook for Systematic Reviews of Interventions and the Preferred Reporting Items for Systematic Reviews and MetaAnalysis.

Ethics and dissemination Ethical approval is not required as this project does not involve primary data collection. We will disseminate our findings through peerreviewed publications and conference presentations. PROSPERO registration number CRD42020157851.

\section{INTRODUCTION}

Health-related quality of life (HRQoL) is a key outcome in dementia care and research. ${ }^{1-3}$ With no dementia cure or disease-modifying treatment available, maximising HRQoL of

\section{Strengths and limitations of this study}

- In contrast to systematic reviews synthesising evidence on multiple health-related quality of life (HRQoL) instruments, our review will investigate in detail the evidence available on one specific instrument to measure dementia-related $\mathrm{HRQOL}$ - the DEMQOL suite of instruments-allowing for a sufficiently detailed analysis of all relevant aspects of the selected instrument.

- We will identify, evaluate and synthesise evidence on the psychometric properties of the DEMQOL suite of instruments, its feasibility, acceptability, appropriateness and on how it was used in research studies-which is a prerequisite to determine its strengths and weaknesses for use in research and care practice, and to identify important research gaps.

- We will apply best practices in conducting systematic reviews, guided by the Cochrane Handbook of Systematic Reviews and the Preferred Reporting Items for Systematic Reviews and Meta-Analyses guidelines.

- We expect that we will not be able to conduct metaanalyses since we likely will not be able to identify a minimum of three studies investigating the same outcome using comparable methods.

people with dementia is the overarching goal of care. ${ }^{4-6}$ Dementia is an umbrella term for a set of progressive, degenerative brain disorders that successively diminish a person's cognitive and functional abilities. Dementia is associated with troubling neuro-psychiatric symptoms, and is, ultimately, fatal. ${ }^{78}$ Currently, 50 million people worldwide are living with dementia ${ }^{9}-500000$ in Canada, ${ }^{10} 5.7$ million in the $\mathrm{USA}^{11}$ and 9.6 million in the EU. ${ }^{12}$ Numbers are expected to be more than triple by $2050 .{ }^{9}$ People with dementia experience decline in physical function and mental health, often associated with poor HRQoL. ${ }^{713}$

Although often used interchangeably, QoL and HRQoL are related but distinct 
concepts. ${ }^{14}$ QoL has been conceptualised as a person's overall general well-being, including physical, material, social and emotional components, rated based on the person's subjective perception (self-report) but may also include objective indicators (eg, observation of someone's behaviour or affect). ${ }^{14}$ QoL is influenced by factors that interact in complex ways: physical health, psychological state, personal beliefs, social relationships and environmental features. ${ }^{1}$ Often terms like 'well-being', 'life satisfaction' or 'comfort' are either used to define QoL, treated as synonymous to QoL or considered similar but distinct concepts. ${ }^{15}$ Authors disagree on whether QoL should be rated purely based on a person's individual perception (self-report) or if it also should include objective indicators (eg, observation of someone's behaviour or affect). ${ }^{14}$ Therefore, it is critical that authors clearly report the definition underlying their research. Our understanding of QoL is based on the WHO's definition of QoL as 'an individual's perception of their position in life in the context of the culture and value systems in which they live and in relation to their goals, expectations, standards and concerns' (The WHOQOL Group, ${ }^{16}$ p1405).

Building on the concept of QoL but narrowing the focus, HRQoL in contrast is defined as an individual's perception of the impact a health condition has on that individual's life. ${ }^{17}$ This is the definition that the DEMQOL suite of instruments ${ }^{18}$ is based on-a set of questionnaires to measure HRQoL in people with dementia. The DEMQOL suite of instruments will be the focus of this review. HRQoL and common dementia symptoms (cognitive and physical impairment and neuro-psychiatric symptoms) are related, but they are not the same. ${ }^{19}$ People with dementia can have good HRQoL despite severe cognitive and physical impairment, and people with mild dementia symptoms can have poor HRQoL. ${ }^{19}$ Therefore, measuring a person's perceptions of how symptoms affect their life (HRQoL), rather than just dementia symptom severity, can provide more specific information about how to best promote well-being in ways that are most meaningful to the person with dementia.

Systematic reviews are available on (a) tools to assess HRQoL in people with dementia in general ${ }^{20}$ or (b) in care homes, ${ }^{3}{ }^{21}$ (c) generic QoL tools for use in care homes $^{22}$ and (d) QoL and HRQoL tools that have been used in clinical trials for interventions targeting people living with dementia or cognitive impairment. ${ }^{23}$ These reviews have identified 34 tools to assess QoL or HRQoL in people with dementia (table 1). Another popular tool not captured in any of these reviews is the interRAI QoL module. ${ }^{24}$ Evidence for reliability and validity for many of these instruments is poor and, in general, there is high heterogeneity in terms of the tools' theoretical foundations, domains measured, and how they apply to different levels of dementia severity. ${ }^{2021}$ It is unclear which of these instruments is most feasible, acceptable or appropriate for use in research and practice. ${ }^{2}$ In line with best practice standards for evaluating the psychometric properties of
Table 1 Overview of tools available to assess QoL or HRQoL in people with dementia

\begin{tabular}{|c|c|}
\hline Acronym & Full name \\
\hline - & Activity and Affect rating scales \\
\hline ACSA & $\begin{array}{l}\text { Anamnestic Comparative Self- } \\
\text { Assessment Scale }\end{array}$ \\
\hline ADRQL & $\begin{array}{l}\text { Alzheimer Disease Related Quality of } \\
\text { Life }\end{array}$ \\
\hline BASQID & $\begin{array}{l}\text { Bath Assessment of Subjective Quality } \\
\text { of Life in Dementia }\end{array}$ \\
\hline- & Byrne-MacLean QoL Index \\
\hline CBS & Cornell-Brown Scale \\
\hline CDQLP & $\begin{array}{l}\text { Community Dementia Quality of Life } \\
\text { Profile }\end{array}$ \\
\hline COOP/WONCA & $\begin{array}{l}\text { Cooperative Functional Health } \\
\text { Assessment Charts/World Organization } \\
\text { of National Colleges, Academies and } \\
\text { Academic Associations of General } \\
\text { Practitioners/Family Physicians }\end{array}$ \\
\hline DCM & Dementia Care Mapping \\
\hline DEMQOL & - \\
\hline DQoL & Dementia Quality of life \\
\hline EQ-5D/EQ-15D & - \\
\hline H.I.L.DE. & $\begin{array}{l}\text { Heidelberg Instrument to assess Quality } \\
\text { of Life in people with dementia }\end{array}$ \\
\hline HUI3 & Health Utilities Index Mark 3 \\
\hline$M C Q$ & Mild Cognitive Impairment Questionnaire \\
\hline OQOLD (A) & $\begin{array}{l}\text { Observing Quality of Life in Dementia } \\
\text { (also a version for advanced }(\mathrm{A}) \\
\text { dementia available) }\end{array}$ \\
\hline PDS & Progressive Deterioration Scale \\
\hline PES-AD & Pleasant Events Schedule \\
\hline PGC-ARS & $\begin{array}{l}\text { Philadelphia Geriatric Center Affect } \\
\text { Rating Scale }\end{array}$ \\
\hline PGCMS & $\begin{array}{l}\text { Philadelphia Geriatric Centre Moral } \\
\text { Scale }\end{array}$ \\
\hline- & $\begin{array}{l}\text { Psychosocial Quality of Life Domains } \\
\text { Measure }\end{array}$ \\
\hline PWB-CIP & $\begin{array}{l}\text { Psychological Well-Being in Cognitively } \\
\text { Impaired Persons }\end{array}$ \\
\hline QLA-P & Quality of Life Assessment-Patient \\
\hline QOL-AD & Quality of Life in Alzheimer's Disease \\
\hline QOL-D & Quality of Life in Dementia \\
\hline QOLAS & Quality of Life Assessment Schedule \\
\hline- & Quality of Life Face Scale \\
\hline QOLS & Quality of Life Scales \\
\hline QUALID & Quality of Life in Late-stage Dementia \\
\hline QUALIDEM & $\begin{array}{l}\text { Quality of life instrument for proxy } \\
\text { completion }\end{array}$ \\
\hline RSOC-QoL & $\begin{array}{l}\text { Resident and Staff Observation } \\
\text { Checklist-Quality of Life }\end{array}$ \\
\hline
\end{tabular}

Continued 


\begin{tabular}{ll} 
Table 1 Continued \\
\hline Acronym & Full name \\
\hline SF-12/SF-36 & - \\
- & Vienna List \\
\hline
\end{tabular}

HRQoL, health-related quality of life.

research tools ${ }^{25}$ we define reliability as statistical measures that indicate how closely two equivalent forms of a tool correlate. Validity, according to these standards, is 'the degree to which evidence and theory support the interpretations of test scores for proposed uses of tests' (AERA et $\left.a l,{ }^{25} \mathrm{p} 11\right)$. Feasibility, acceptability and appropriateness are implementation outcomes-that is, outcomes that reflect tool users' experiences with using the tool and their perception of whether the tool can and should be used in the future. ${ }^{26}$ We provide detailed definitions and operationalisations of each of these terms in the methods section (inclusion/exclusion criteria).

Previous reviews have attempted to give an overview of measurement properties and usability across and between QoL tools. However, none sufficiently analyse all relevant aspects to understand (a) a certain tool's conceptual characteristics, (b) whether that tool is psychometrically sound, feasible, acceptable and appropriate for use in research and practice and (c) how that tool has been used in research as of now. Therefore, we believe that systematic reviews examining one selected QoL tool in detail are needed.

In this review, we chose to focus on the DEMQOL suite of instruments ${ }^{18}$ for the following reasons. First, the DEMQOL suite is specifically designed to measure HRQoL among people with dementia. Generic QoL tools (eg, EQ5D, ${ }^{27}$ SF-12, ${ }^{28}$ interRAI QoL module ${ }^{24}$ often work poorly to capture the perspective of people with dementia. ${ }^{18}$ Second, among the available instruments to measure HRQoL in people with dementia, the DEMQOL suite is considered one of the best given its relatively strong theoretical foundations and psychometric properties (table 2). ${ }^{2}$ The DEMQOL and DEMQOL-Proxy were developed based on robust theory and a rigorous process of tool development that included (a) a review of available conceptualisations of QoL and HRQoL, (b) a review of available measures of HRQoL in dementia, (c) qualitative interviews with people with dementia and their families and (d) the development of a conceptual framework for dementia-related HRQoL. ${ }^{18}{ }^{29}$ Therefore content validity is acceptable. In their review, Bowling et $a l^{2}$ report evidence for acceptability and feasibility of the DEMQOL and DEMQOL-Proxy. Evidence is also available on convergent and discriminant validity. ${ }^{2}$ Evidence on the tools' factor structure, responsiveness and respondent burden is limited. ${ }^{2}$ No evidence is available on known group differences and on psychometric properties of cultural and language adaptations of these tools. ${ }^{2}$ The DEMQOL-CH is based on the DEMQOL-Proxy with similar findings related to its reliability and validity. ${ }^{30}$ Third, the DEMQOL and its variations (proxy versions, preference-based indices for use in economic evaluation and translations into various languages; table 2$)^{31}$ are among the most popular instruments to measure HRQoL in research with people with dementia. As of 23 May 2020, the developers had documented 89 studies that used the DEMQOL suite of instruments. ${ }^{32}$ Fourth, with the DEMQOL-CH, ${ }^{30}$ a version is now available that can be completed by staff caring for residents with dementia living in congregate care settings such as nursing homes or assisted/supportive living. This is important because the majority of these residents have dementia that is severe enough to limit their ability to self-report, ${ }^{33-38}$ and often residents do not have a family/friend carer who visits and who could provide a proxy assessment. ${ }^{39}$ A tool that can be completed by care staff in a way that is reliable, valid, feasible, acceptable and appropriate opens the possibility of routine HRQoL assessment-an important prerequisite for improving residents' HRQoL.

No dementia-specific QoL or HRQoL tool has been rigorously and comprehensively assessed for reliability, validity, feasibility, acceptability, appropriateness and use in research, using a comprehensive review of the literature. Therefore, focusing on the DEMQOL suite of instruments, in this review we will answer the following research questions:

1. How has the DEMQOL system been used in research?

a. What research questions did studies using the DEMQOL system investigate?

b. Which study settings and populations did studies using the DEMQOL system focus on?

c. What is the quality of the research using the DEMQOL system?

2. How has the DEMQOL system been evaluated?

a. What evidence is available on the development of the DEMQOL system?

b. What are the psychometric properties of the DEMQOL system?

c. What is the evidence on the DEMQOL system's feasibility, acceptability and appropriateness?

\section{METHODS AND ANALYSIS \\ Review design}

We will conduct a systematic mixed methods synthesis of research. ${ }^{40}$ Our review methods and presentation of results will follow the Cochrane Handbook of Systematic Reviews of Interventions ${ }^{41}$ and the Preferred Reporting Items for Systematic Reviews and Meta-Analyses (PRISMA) guidelines. ${ }^{42}$ This paper follows the PRISMA-P reporting guidelines for systematic review protocols. ${ }^{43} \mathrm{We}$ started the review in Jan 2019. Currently, we are finalising the screening of full texts. The review is scheduled to be completed by March 2021.

\section{Patient and public involvement}

This systematic review is part of a larger research programme on routinely measuring and improving 
Table 2 Overview of DEMQOL versions and their characteristics

\begin{tabular}{|c|c|c|c|c|c|}
\hline & DEMQOL & DEMQOL-Proxy & DEMQOL-U & DEMQOL-Proxy-U & DEMQOL-CH \\
\hline Year of publication & 2005 & 2005 & 2013 & 2013 & 2019 \\
\hline Target group & $\begin{array}{l}\text { Persons with } \\
\text { mild to moderate } \\
\text { dementia (MMSE } \\
\geq 10 \text { ) }\end{array}$ & $\begin{array}{l}\text { Persons with all stages } \\
\text { of dementia (up to } \\
\text { severe) }\end{array}$ & Same as DEMQOL & $\begin{array}{l}\text { Same as DEMQOL- } \\
\text { Proxy }\end{array}$ & $\begin{array}{l}\text { Persons with } \\
\text { all stages of } \\
\text { dementia (up to } \\
\text { severe) }\end{array}$ \\
\hline $\begin{array}{l}\text { Mode of } \\
\text { administration }\end{array}$ & $\begin{array}{l}\text { Interview of } \\
\text { person with } \\
\text { dementia }\end{array}$ & $\begin{array}{l}\text { Interview of proxy of } \\
\text { person with dementia }\end{array}$ & $\begin{array}{l}\text { NA (DEMQOL } \\
\text { scores are used } \\
\text { and turned into } \\
\text { preference-based } \\
\text { (utility) values) }\end{array}$ & $\begin{array}{l}\text { NA (DEMQOL-Proxy } \\
\text { scores are used } \\
\text { and turned into } \\
\text { preference-based } \\
\text { (utility) values) }\end{array}$ & $\begin{array}{l}\text { Completed by } \\
\text { care staff proxy } \\
\text { of person with } \\
\text { dementia }\end{array}$ \\
\hline Number of items & 28 & 31 & $\begin{array}{l}5 \text { (selected } 1 \\
\text { item out of each } \\
\text { identified domain) }\end{array}$ & $\begin{array}{l}4 \text { (selected } 1 \text { item } \\
\text { out of each identified } \\
\text { domain, other than } \\
\text { daily activities) }\end{array}$ & 31 \\
\hline Scoring & $\begin{array}{l}\text { Items are scored } \\
\text { on a 4-point Likert } \\
\text { scale ranging } \\
\text { from } 1 \text { to } 4 ; \\
\text { Positive items are } \\
\text { scored reversely } \\
\text { so lower scores } \\
\text { always indicate } \\
\text { worse HRQoL; } \\
\text { item scores are } \\
\text { summed (possible } \\
\text { range 28-112) }\end{array}$ & $\begin{array}{l}\text { Items are scored on } \\
\text { a } 4 \text {-point Likert scale } \\
\text { ranging from } 1 \text { to } 4 ; \\
\text { Positive items are } \\
\text { scored reversely so } \\
\text { lower scores always } \\
\text { indicate worse } \\
\text { HRQoL; item scores } \\
\text { are summed (possible } \\
\text { range } 31-124)\end{array}$ & $\begin{array}{l}\text { Based on a health } \\
\text { state classification } \\
\text { system and } \\
\text { population-based } \\
\text { preference values, } \\
\text { a score between } 0 \\
\text { (death) and } 1 \text { (full } \\
\text { health) is generated }\end{array}$ & $\begin{array}{l}\text { Based on a health } \\
\text { state classification } \\
\text { system and } \\
\text { population-based } \\
\text { preference values, } \\
\text { a score between } 0 \\
\text { (death) and } 1 \text { (full } \\
\text { health) is generated }\end{array}$ & $\begin{array}{l}\text { Items are scored } \\
\text { on a 4-point Likert } \\
\text { scale ranging } \\
\text { from } 1 \text { to 4; } \\
\text { Positive items are } \\
\text { scored reversely } \\
\text { so lower scores } \\
\text { always indicate } \\
\text { worse HRQoL; } \\
\text { item scores are } \\
\text { summed (possible } \\
\text { range 31-124) }\end{array}$ \\
\hline Test-retest & $\mathrm{ICC}=0.76$ & $\mathrm{ICC}=0.67-0.84$ & NA & NA & $\mathrm{ICC}=0.72$ \\
\hline Utility scores & NA & NA & $0.243-0.986$ & $0.363-0.937$ & NA \\
\hline Validity & $\begin{array}{l}\text { Correlations } \\
\text { with QOL-AD } \\
\text { scores }(r=0.54) \\
\text { and DQOL items } \\
(r=0.29-0.45)\end{array}$ & $\begin{array}{l}\text { Correlations with QOL- } \\
\text { AD-caregiver scores } \\
(r=0.52)\end{array}$ & & & $\begin{array}{l}\text { Correlations with } \\
\text { DCM scores } \\
(r=0.34-0.67)\end{array}$ \\
\hline
\end{tabular}

DCM, Dementia Care Mapping; DQoL, Dementia Quality of Life; HRQoL, health-related quality of life; ICC, Intraclass correlation; MMSE, Mini Mental State Examination; QOL-AD, Quality of Life in Alzheimer's Disease.

the HRQoL in people with dementia living in congregate settings. On 09 October 2019, our research team convened a policy-level forum on QoL in the Canadian province of Alberta, ${ }^{44}$ including health systems level and health ministry level key decision makers, representatives from care organisations, people with dementia and their family/friend caregivers. The purpose of the forum was to develop a framework for improving QoL for persons with moderate to severe dementia living in congregate care settings. Perspectives of people with dementia and their family/friend caregivers were central throughout all discussions. Two key outcomes of this forum included: (a) a mandate to conduct this systematic review in order to further explore suitability of the DEMQOL suite of instruments for routine use in congregate care settings, and (b) formation of a QoL workgroup to further advance the QoL work started by our team. This workgroup includes representatives of all stakeholder groups involved in the QoL forum and oversees the various activities of our team, including this systematic review. We will feed back results of this review to the QoL workgroup and to the larger team on an ongoing basis, and this review will inform 
further research projects and activities to improve QoL of people with dementia living in congregate care settings.

\section{Search strategy}

Supported by a scientific librarian, we will search the databases MEDLINE, EMBASE, PsycInfo, Journals@ovid, CINAHL, Abstracts in Social Gerontology, Academic Search Complete, Cochrane Library, Scopus, Web of Science, ProQuest Dissertations \& Theses Global Google Scholar and Science Direct. We will search the terms DEMQOL or DEM-QOL or Dementia Quality of Life scale in the database default fields including title, abstract, $\mathrm{MeSH} /$ subject heading and author-supplied keyword fields, as well as, in the fulltext of records (online supplemental appendix 1). We will not limit our search based on language and year of publication, and we will search the time frame covered by the data bases. We will search reference lists of all included studies for additional references.

\section{Data management}

We will manage references using Rayyan ${ }^{45}$-a free reference management software designed for literature reviews that facilitates online collaboration and blinding of reviewers during screening activities. All references including abstracts will be uploaded to Rayyan and title/ abstract and fulltext screening will be done using this software. All team members will receive training on the application of Rayyan prior to the screening, and we will conduct regular meetings and calibration exercises to improve application of the inclusion and exclusion criteria.

\section{Inclusion and exclusion criteria}

Our primary inclusion criterion (table 3 ) is whether the study either (a) reports on the development, validation or assessment of feasibility, acceptability or appropriateness of any of the DEMQOL versions available or (b) used any of the DEMQOL versions to assess study outcomes. Original studies of any design or systematically conducted reviews are eligible. If the search specified above identifies non-peer reviewed references (grey literature), we will include these references if they meet our inclusion criteria. We will include studies regardless of the country of origin, publication language, study setting or population. Languages spoken among members of our study team include: Chinese, English, French, German, Nepalese and Urdu. Our networks include colleagues who speak Danish, Dutch, Farsi, Italian, Norwegian, Portuguese, Spanish and Swedish, who will help us to assess eligibility of studies in these languages. Should we encounter studies with no English abstract in languages other than those listed, we will further leverage our networks to find a colleague who speaks this language. We have successfully applied this approach in previous literature reviews. ${ }^{46}{ }^{47}$ Studies that assessed HRQoL as a study outcome, using either of the DEMQOL instruments will be included regardless of the research question(s) and regardless of whether HRQoL was the main study outcome (dependent variable), an independent variable (predictor) or a covariate to adjust models.

\section{Study screening}

After removal of duplicates, team member pairs will independently screen titles and abstracts of retrieved references. Discrepancies will be discussed in the group and resolved by consensus. Full texts will be retrieved for included references and for references with insufficient information in the title/abstract to decide on inclusion. Full text screening will follow the same method as title/ abstract screening.

\section{Quality appraisal}

To assess the risk of bias of each included study assessing the reliability or validity of one of the DEMQOL tools, we will use the validated COSMIN risk of bias checklist for systematic reviews of Patient-Reported Outcome Measures. ${ }^{48}$ To assess the risk of bias of each other included study, we will use one of four validated checklists, as appropriate for the respective study design:

- Systematically conducted reviews: Assessment of Multiple Systematic Reviews (AMSTAR) tool. ${ }^{4-52}$

- Clinical studies with or without a control group and with or without randomised allocation of participants: Quality Assessment Tool for Quantitative Studies (QATQS). ${ }^{5354}$

- Cross-sectional studies: Estabrooks' Quality Assessment and Validity Tool for Cross-Sectional Studies, which is based on established criteria for assessing quality of research studies. ${ }^{55} 56$

- Qualitative studies: Critical Appraisal Skills Programme (CASP) Qualitative Research Checklist. ${ }^{57}$

Studies will be assessed independently by two team members and discrepancies resolved by consensus. We will score overall quality of each study, using a method we have previously used in various systematic reviews. ${ }^{58-62}$ As per the developer of this method, ${ }^{63}$ we will calculate the ratio of the obtained score to the maximum possible score for each study (possible range: $0-1$ ). The maximum possible score varies depending on the checklist used and the number of checklist items applicable. We will rank studies as weak $(\leq 0.50)$, low moderate $(0.51-0.66)$, high moderate $(0.67-0.79)$ or strong $(\geq 0.80)$. We will also summarise and describe the key areas of weakness for all studies within each type of research design.

\section{Data extraction}

Our study team collaboratively adapted and pretested data extraction templates (online supplemental appendix 2), successfully used in previous systematic reviews. ${ }^{46} 47$ One team member will extract study details into the template, and a second team member will double check the extracted information and discrepancies will be resolved by consensus. We will extract:

- First author

- Year of publication

- Title 


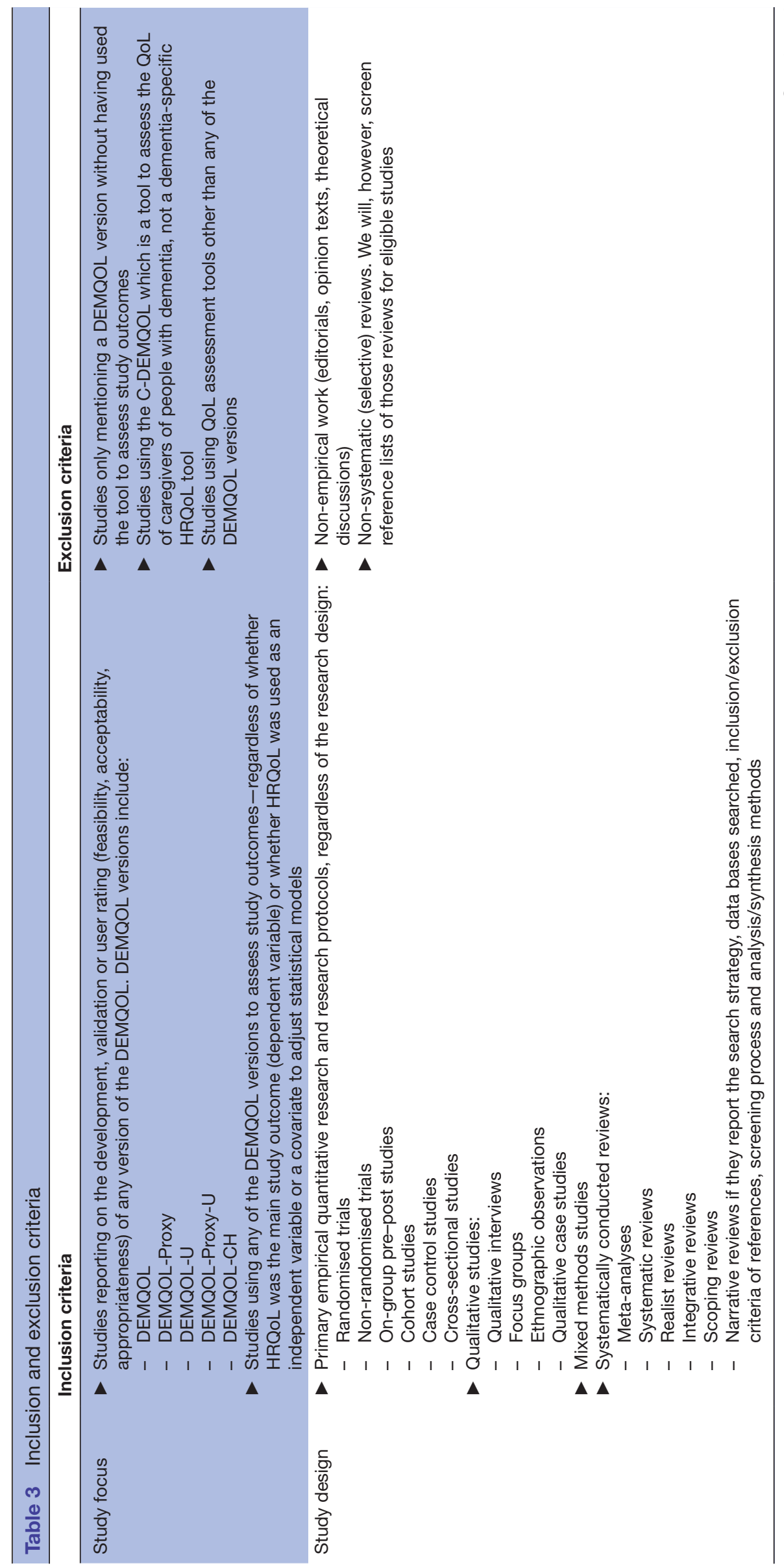




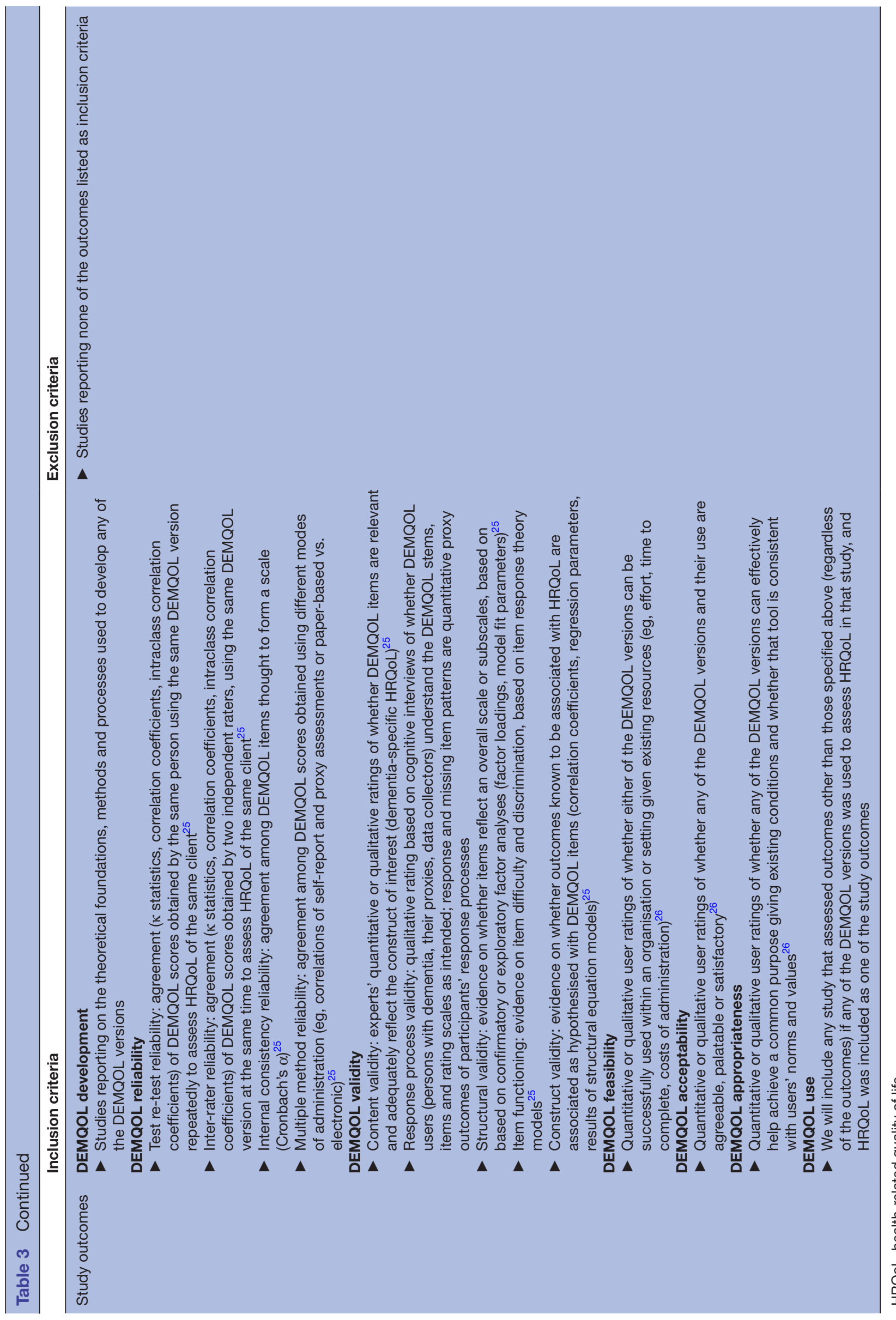


- Journal name (or type of reference such as thesis, report, textbook)

- Country of study

- Study aim(s), goal(s), purpose(s) or question(s) and which of our review questions these refer to (ie, development of the DEMQOL; assessments of its reliability and/or validity; assessments of its feasibility, acceptability, appropriateness; use of the DEMQOL as dependent study outcome or as covariate

- Study design

- Study setting and sample

- DEMQOL version(s) used

- Dependent study variables and how they were measured (if applicable)

- Independent study variables and how they were measured (if applicable)

- Main results as they relate to the development of either of the DEMQOL versions; DEMQOL reliability, validity; DEMQOL feasibility, acceptability, appropriateness; DEMQOL use (operationalised as per table 3).

\section{Contacting authors for additional details}

If a study does not report enough details, we will contact the study authors by email and invite them to clarify or add information to inform inclusion or exclusion of this study, risk for bias assessments and/or data extractions. In the case of non-response, we will send out reminders after 7, 10 and 13 days.

\section{Analyses}

To address research question 1 , we will first conduct a thematic analysis ${ }^{64}$ of narrative data (eg, types of research questions asked) from the studies that used the DEMQOL to assess research outcomes, converting narrative to categorical data. Using figures and tables, we will descriptively present the number and proportion of studies that represent each category—-for example, DEMQOL version used, types of research questions asked, participant groups included, country of origin, study setting, study design, risk for bias category and so on.

To address research question 2, we will use descriptive statistics and narrative synthesis to summarise the proportion of studies that have assessed each of the elements outlined in table 3 (development, reliability, validity, feasibility, acceptability, appropriateness of any of the DEMQOL versions), and the range of results reported by these studies. We will operationalise these results as per table 3 and report them by DEMQOL version used.

For qualitative results, we will conduct a content analysis of the key themes and supporting data related to the respective outcome and whether the content of these themes varied across studies. For quantitative results, we will report the range of scores, and the number and proportion of studies reporting statistically significant positive associations, statistically negative associations and statistically non-significant associations for a certain study outcome (vote counting). We will not attempt to synthesise study findings statistically (meta-analyses) since our research questions are descriptive, overall effect sizes across studies are not part of our two research questions, and study variables and populations are likely to be heterogenous enough that meta-analysis would not be appropriate.

\section{ETHICS AND DISSEMINATION}

Ethics approval will not be needed for this study as we will not collect primary data from individuals or organisations. Data of studies included in this systematic review cannot be linked to individuals or organisations. We intend to publish findings of the review in a peer-reviewed journal (will be made available on the DEMQOL website) and present findings at an international peer-reviewed conference. We will prepare a lay summary of the findings for knowledge users on what is known about the DEMQOL suite of instruments, and recommendations for use in practice. Results of this review will synthesise information on how DEMQOL has been used and how its psychometric properties have been described or evaluated in various studies, which will enable researchers who want to use DEMQOL tool in future to evaluate its psychometric properties.

Acknowledgements We would like to thank the decision makers, representatives of care organisations, people with dementia and their family/friend caregivers who attended our policy-level forum on QoL for their valuable inputs, the lively discussions and their ongoing support. Their suggestions substantially informed this systematic review.

Contributors $\mathrm{MH}, \mathrm{CAE}, \mathrm{SAC}, \mathrm{HM}$, SB and LH developed the research question, the systematic review design and planned and designed the study protocol. $\mathrm{MH}$ is leading the systematic review project. MH in collaboration with a scientific librarian developed and tested the search strategy. Guided by MH and SAC, BE, SS, RD, TT and $\mathrm{JL}$ tested and refined the search strategy and adapted the screening and data extraction templates. All authors critically read and commented on the manuscript and approved its submission.

Funding This research has been supported by MH's Faculty of Nursing, University of Alberta Professorship in Continuing Care Policy Research. CAE holds a Tier I Canada Research Chair in Knowledge translation that has also partly supported this work. None of the funders has played any role in developing the systematic review protocol.

Competing interests None declared.

Patient consent for publication Not required.

Supplemental material This content has been supplied by the author(s). It has not been vetted by BMJ Publishing Group Limited (BMJ) and may not have been peer-reviewed. Any opinions or recommendations discussed are solely those of the author(s) and are not endorsed by BMJ. BMJ disclaims all liability and responsibility arising from any reliance placed on the content. Where the content includes any translated material, BMJ does not warrant the accuracy and reliability of the translations (including but not limited to local regulations, clinical guidelines, terminology, drug names and drug dosages), and is not responsible for any error and/or omissions arising from translation and adaptation or otherwise.

Open access This is an open access article distributed in accordance with the Creative Commons Attribution Non Commercial (CC BY-NC 4.0) license, which permits others to distribute, remix, adapt, build upon this work non-commercially, and license their derivative works on different terms, provided the original work is properly cited, appropriate credit is given, any changes made indicated, and the use is non-commercial. See: http://creativecommons.org/licenses/by-nc/4.0/.

ORCID iDs

Matthias Hoben http://orcid.org/0000-0003-3465-315X 
Laura Hughes http://orcid.org/0000-0001-9530-7053

\section{REFERENCES}

1 Kwasky AN, Harrison BE, Whall AL. Quality of life and dementia: an integrated review of literature. Alzheimer's Care Today 2010;11:186-95.

2 Bowling A, Rowe G, Adams S, et al. Quality of life in dementia: a systematically conducted narrative review of dementia-specific measurement scales. Aging Ment Health 2015;19:13-31.

3 Robertson S, Cooper C, Hoe J, et al. Proxy rated quality of life of care home residents with dementia: a systematic review. Int Psychogeriatr 2017;29:569-81.

4 Livingston G, Sommerlad A, Orgeta V, et al. Dementia prevention, intervention, and care. Lancet 2017;390:2673-734.

5 Pickett J, Bird C, Ballard C, et al. A roadmap to advance dementia research in prevention, diagnosis, intervention, and care by 2025 . Int J Geriatr Psychiatry 2018;33:900-6.

6 van der Steen JT, Radbruch L, Hertogh CMPM, et al. White paper defining optimal palliative care in older people with dementia: a Delphi study and recommendations from the European association for palliative care. Palliat Med 2014;28:197-209.

7 Haaksma ML, Leoutsakos J-MS, Bremer JAE, et al. The clinical course and interrelations of dementia related symptoms. Int Psychogeriatr 2018:30:859-66.

$8 \mathrm{Kua} \mathrm{EH}, \mathrm{Ho} \mathrm{E}$, Tan $\mathrm{HH}$, et al. The natural history of dementia. Psychogeriatrics 2014;14:196-201.

9 Alzheimer's Disease International. World Alzheimer report 2018: the state of the art of dementia research. London New frontiers; 2018.

10 Alzheimer Society of Canada. Understanding dementia: 2017-2018 impact report; 2018.

11 Alzheimer's Association. 2018 Alzheimer's disease facts and figures. Alzheimer's \& Dementia 2018;14:367-429.

12 OECD, European Union. Health at a glance: Europe 2016 - state of health in the EU cycle. Paris OECD Publishing; 2016.

13 Martyr A, Nelis SM, Quinn C, et al. Living well with dementia: a systematic review and correlational meta-analysis of factors associated with quality of life, well-being and life satisfaction in people with dementia. Psychol Med 2018;48:2130-9.

14 Karimi M, Brazier J. Health, health-related quality of life, and quality of life: what is the difference? Pharmacoeconomics 2016;34:645-9.

15 Pinto S, Fumincelli L, Mazzo A, et al. Comfort, well-being and quality of life: discussion of the differences and similarities among the concepts. Porto Biomed J 2017;2:6-12.

16 The WHOQOL Group. The world Health organization quality of life assessment (WHOQOL): position paper from the world Health organization. Soc Sci Med 1995;41:1403-9.

17 Bullinger M, Anderson R, Cella D, et al. Developing and evaluating cross-cultural instruments from minimum requirements to optimal models. Qual Life Res 1993;2:451-9.

18 Smith S, Lamping D, Banerjee S, et al. Measurement of healthrelated quality of life for people with dementia: development of a new instrument (DEMQOL) and an evaluation of current methodology. Health Technol Assess 2005;9:1-93.

19 Banerjee S, Smith SC, Lamping DL, et al. Quality of life in dementia: more than just cognition. An analysis of associations with quality of life in dementia. J Neurol Neurosurg Psychiatry 2006;77:146-8.

20 Dichter MN, Schwab CGG, Meyer G, et al. Linguistic validation and reliability properties are weak investigated of most dementia-specific quality of life measurements-a systematic review. J Clin Epidemiol 2016;70:233-45.

21 Hughes LJ, Farina N, Page TE, et al. Psychometric properties and feasibility of use of dementia specific quality of life instruments for use in care settings: a systematic review. Int Psychogeriatr 2019:1-15 (published Online First: 2019/01/04).

22 Aspden T, Bradshaw SA, Playford ED, et al. Quality-Of-Life measures for use within care homes: a systematic review of their measurement properties. Age Ageing 2014;43:596-603.

23 Yang F, Dawes P, Leroi I, et al. Measurement tools of resource use and quality of life in clinical trials for dementia or cognitive impairment interventions: a systematically conducted narrative review. Int J Geriatr Psychiatry 2018;33:e166-76.

24 interRAl. Self-Reported quality of life (QOL). Available: https://www. interrai.org/quality-of-life.html [Accessed 15 Aug 2019].

25 AERA, APA, NCME. Standards for educational and psychological testing. 6th ed. Washington, DC, 2014

26 Proctor E, Silmere H, Raghavan R, et al. Outcomes for implementation research: conceptual distinctions, measurement challenges, and research agenda. Adm Policy Ment Health 2011;38:65-76.
27 EQ-5D. Available: https://euroqol.org/2020-02-27

28 12-Item short form survey (SF-12). Available: https://www.rand.org/ health-care/surveys_tools/mos/12-item-short-form.html [Accessed 27 Feb 2020].

29 Smith SC, Lamping DL, Banerjee S, et al. Development of a new measure of health-related quality of life for people with dementia: DEMQOL. Psychol Med 2007;37:737-46.

30 Hughes LJ, Farina N, Page TE, et al. Adaptation of the DEMQOLProxy for routine use in care homes: a cross-sectional study of the reliability and validity of DEMQOL-CH. BMJ Open 2019;9:e028045.

31 DEMQOL: dementia quality of life measure. Available: https://www. bsms.ac.uk/research/neuroscience/cds/research/demqol.aspx [Accessed 31 Mar 2019]

32 Feeney Y, Russell A, Farina N. The DEMQOL bibliography. Zenodo2020.

33 Alzheimer's Association. 2020 Alzheimer's disease facts and figures. Alzheimer's \& Dementia 2020;16:391-460.

34 Canadian Institute for Health Information (CIHI). Dementia in longterm care, 2019. Available: https://www.cihi.ca/en/dementia-incanada/dementia-across-the-health-system/dementia-in-long-termcare [Accessed 23 Jul 2019].

35 Bartfay E, Bartfay WJ, Gorey KM. Prevalence and correlates of potentially undetected dementia among residents of institutional care facilities in Ontario, Canada, 2009-2011. Int J Geriatr Psychiatry 2013;28:1086-94.

36 Maxwell CJ, Amuah JE, Hogan DB, et al. Elevated hospitalization risk of assisted living residents with dementia in Alberta, Canada. J Am Med Dir Assoc 2015;16:568-77.

37 Zimmerman S, Sloane PD, Reed D. Dementia prevalence and care in assisted living. Health Aff 2014;33:658-66

38 Prince M, Knapp M, Guerchet M. Dementia UK: update. London: Alzheimer's Society, 2014.

39 Chamberlain S, Baik S, Estabrooks C. Going it alone: a scoping review of Unbefriended older adults. Can J Aging 2018;37:1-11.

40 Harden A. Mixed-Methods systematic reviews: integrating quantitative and qualitative findings 2010:1-8.

41 Higgins JPT, Green S, eds. Cochrane handbook for systematic review of interventions versions 5.1.0. The Cochraine collaboration, 2011.

42 Moher D, Liberati A, Tetzlaff J, et al. Preferred reporting items for systematic reviews and meta-analyses: the PRISMA statement. PLoS Med 2009;6:e1000097.

43 Shamseer L, Moher D, Clarke M, et al. Preferred reporting items for systematic review and meta-analysis protocols (PRISMA-P) 2015 elaboration and explanation. BMJ2015;349:g7647.

44 Translating Research in Elder Care (TREC). Policy forum on quality of life for residents living with dementia, 2020. Available: https:// trecresearch.ca/download/documents/policy_forum_on_quality_ of_life_report/trec_qol_policy_forum_report_17jan2020pdf;v1? attachment $=1$ [Accessed 08 Oct 2020].

45 Qatar Computing Research Institute. Rayyan QCRI. Available: https://rayyan.qcri.org/welcome [Accessed 04 Oct 2019].

46 Hoben M, Clarke A, Huynh KT, et al. Barriers and facilitators in providing oral care to nursing home residents, from the perspective of care aides: a systematic review and meta-analysis. Int J Nurs Stud 2017;73:34-51.

47 Hoben M, Kent A, Kobagi N, et al. Effective strategies to motivate nursing home residents in oral care and to prevent or reduce responsive behaviors to oral care: a systematic review. PLoS One 2017;12:e0178913.

48 Mokkink LB, de Vet HCW, Prinsen CAC, et al. COSMIN risk of bias checklist for systematic reviews of patient-reported outcome measures. Qual Life Res 2018;27:1171-9.

49 Shea BJ, Grimshaw JM, Wells GA, et al. Development of AMSTAR: a measurement tool to assess the methodological quality of systematic reviews. BMC Med Res Methodol 2007;7:10.

50 Shea BJ, Bouter LM, Peterson J, et al. External validation of a measurement tool to assess systematic reviews (AMSTAR). PLoS One 2007;2:e1350.

51 Shea BJ, Hamel C, Wells GA, et al. AMSTAR is a reliable and valid measurement tool to assess the methodological quality of systematic reviews. J Clin Epidemiol 2009;62:1013-20.

52 Kang D, Wu Y, Hu D, et al. Reliability and external validity of AMSTAR in assessing quality of TCM systematic reviews. Evid Based Complement Alternat Med 2012;2012:1-7.

53 Thomas $\mathrm{BH}$, Ciliska D, Dobbins $\mathrm{M}$, et al. A process for systematically reviewing the literature: providing the research evidence for public health nursing interventions. Worldviews Evid Based Nurs 2004;1:176-84.

54 Armijo-Olivo S, Stiles CR, Hagen NA, et al. Assessment of study quality for systematic reviews: a comparison of the Cochrane collaboration risk of bias tool and the effective public health practice 
project quality assessment tool: methodological research. J Eval Clin Pract 2012;18:12-18.

55 Clarke M, Oxman AD, eds. Cochrane reviewer's handbook 4.1.4. The Cochrane Library: Oxford, 2001.

56 Kmet L, Lee R, Cook L. Standard quality assessment criteria for evaluating primary research papers from a variety of fields, 2004. Available: https://www.ihe.ca/publications/standard-qualityassessment-criteria-for-evaluating-primary-research-papers-from-avariety-of-fields

57 (CASP) CASP. CASP (Qualitative) checklists 2018 [Available from. Available: https://casp-uk.net/wp-content/uploads/2018/03/CASPQualitative-Checklist-2018_fillable_form.pdf [Accessed 24 June, 2019].

58 Kajermo KN, Boström A-M, Thompson DS, et al. The barriers scale-the barriers to research utilization scale: a systematic review. Implement Sci 2010;5:32.

59 Squires JE, Estabrooks CA, Gustavsson P, et al. Individual determinants of research utilization by nurses: a systematic review update. Implement Sci 2011;6:1.
60 Squires JE, Hutchinson AM, Boström A-M, et al. To what extent do nurses use research in clinical practice? A systematic review. Implementation Sci 2011;6.

61 Squires JE, Hoben M, Linklater S, et al. Job satisfaction among care aides in residential long-term care: a systematic review of contributing factors, both individual and organizational. Nurs Res Pract 2015;2015:1-24.

62 Hoben M, Berendonk C, Buscher I. Scoping review of nursing-related dissemination and implementation research in German-speaking countries: mapping the field/Scoping review. Int $\mathrm{J}$ Health Prof 2014;1:34-49.

63 de Vet HCW, de Bie RA, van der Heijden GJMG, et al. Systematic reviews on the basis of methodological criteria. Physiotherapy 1997;83:284-9.

64 Thomas J, Harden A. Methods for the thematic synthesis of qualitative research in systematic reviews. BMC Med Res Methodol 2008;8:45. 